\title{
Gestão de custos em organizações hospitalares: sistemática por centro de custos
}

\section{Cost management in hospital organizations: systematics by cost center}

\author{
Antonio Augusto Brion Cardoso ${ }^{1}$, Larissa Marocco Souza ${ }^{2}$, \\ Anderson de Oliveira Reis ${ }^{3}$, Vinícius Masson Palha ${ }^{4}$
}

Resumo

\begin{abstract}
A gestão de custos em qualquer tipo de organização tem se tornado fundamental nos dias de hoje. Para tanto, objetivou-se com este estudo apresentar como a gestão de custo hospitalar, por meio do método de custeio por absorção e por centros de custos, realiza a apuração dos custos em um hospital de grande porte na Zona da Mata mineira. Para alcançar este objetivo, foi realizada uma pesquisa descritiva e qualitativa, adotando a ferramenta de estudo de caso como forma de observar as práticas de gestão de custos adotadas, além da análise documental e observação direta no ambiente estudado. A partir da pesquisa, foi possível identificar os procedimentos de apuração de custos, e concluir que a empresa estudada apresenta um sistema de custo eficiente e bem estruturado, auxiliando no alcance dos objetivos, tomada de decisão e na administração dos recursos financeiros.
\end{abstract}

Palavras-chave: Hospitais. Método de custeio. Centro de custo.

\begin{abstract}
Cost management in any type of organization has become fundamental today. Therefore, the objective of this study was to present how hospital cost management, through the absorption costing method and cost centers, calculates costs in a large hospital in Zona da Mata, Minas Gerais. To achieve this objective, a descriptive and qualitative research was carried out, adopting the case study tool as a way of observing the adopted cost management practices, in addition to documentary analysis and direct observation in the studied environment. From the research, it was possible to identify the procedures for calculating costs, and conclude that the studied company has an efficient and well-structured cost system, helping to achieve the objectives, decision making and the administration of financial resources.
\end{abstract}

Keywords: Hospitals. Costing method. Cost center.

\footnotetext{
${ }^{1}$ Doutorando em Administração na Universidade Federal do Espírito Santo (Ufes), Espírito Santo, Brasil. Professor da Universidade Federal de Juiz de Fora (UFJF), campus Governador Valadares, Minas Gerais, Brasil. E-mail: gutobrion@gmail.com

${ }^{2}$ Especialista em Controladoria e Finanças pela Faculdade Metodista Granbery. E-mail: larissasouzaadm@hotmail.com

${ }^{3}$ Doutorando em Administração na Universidade Federal de Viçosa (UFV), Minas Gerais, Brasil. Professor da Universidade Federal de Juiz de Fora, campus Governador Valadares, Minas Gerais, Brasil. E-mail: andersonccoufv@gmail.com

${ }^{4}$ Mestrado em Economia pela Universidade Candido Mendes (UCAM). Professor do Centro Universitário do Sudeste Mineiro (Unicsum), Minas Gerais, Brasil. E-mail: vinicius.masson.palha@hotmail.com
} 


\section{Introdução}

No final do século XX, Baumgartner (1998) profetizou afirmando que a era do empirismo na gestão hospitalar estava com seus dias contados. O fato é que as mudanças ocorridas nos mercados nos últimos anos têm exigido de todas as empresas desempenhos cada vez melhores para se manterem vivas e competitivas. O uso de ferramentas de gestão que facilitem, ou que otimizem os processos de planejamento e controle, tem se tornado indispensável nos dias atuais em qualquer tipo de organização, seja ela pequena ou grande, prestadora de serviço ou indústria.

Sobre as instituições da área de saúde, vale ressaltar que elas diferem das demais empresas de serviços, pelo seu grau de complexidade e a variedade de atividades exercidas. Segundo Borba (1991), um hospital é uma empresa de alta complexidade, tanto nas influências conjunturais sujeito às contingências econômico-sociais, quanto nos seus aspectos orgânico-estruturais, devendo manter-se como prestador de serviços assistenciais à comunidade, buscando promover a saúde dentro de um bom padrão técnico e em um ótimo nível de humanização nas suas atividades operacionais.

As palavras de Baumgartner (1998) se fazem mais claras atualmente no Brasil, onde nota-se uma escassez de recursos financeiros para serem investidos em saúde; sendo assim, os gestores precisam buscar um meio de otimizar os recursos com a finalidade de não comprometer os serviços assistenciais da entidade. Como forma de minimizar essa situação, torna-se imprescindível uma administração profissional; daí o grande número de gestores com formação na área, compondo o quadro de executivos nestas instituições.

Assim como qualquer organização, as atividades hospitalares necessitam ser controladas e bem planejadas, tudo isso com o propósito de garantir o resultado positivo ao final do exercício; desta forma, ter uma boa gestão de custos tornase indispensável para se otimizar os recursos. Bonacin e Araújo (2010) afirmam que a eficiência e a eficácia gerencial passam pela gestão de custos, ou seja, para eles, a excelência hospitalar tem relação direta com eficácia em custos e com a qualidade do serviço prestado.

Dallora (2007) destaca que infelizmente muitos gestores não possuem o conhecimento necessário acerca dos custos hospitalares, sendo assim, as informações que são disponibilizadas sobre o assunto são pouco aproveitadas. Souza et al. (2013) corroboram afirmando que a área de saúde no Brasil é a mais rica em termos de desenvolvimento de teoria de custos, por outro lado é uma das mais carentes de aplicação desses conhecimentos.

Sobre os custos hospitalares, Negra e Negra (2001) afirmam que estes devem ser um instrumento de trabalho fundamental para a otimização das operações do hospital, alertando a administração para quaisquer resultados que exijam correção, auxiliando assim nas decisões de investimentos em imobilizado, na expansão de instalações hospitalares, na definição dos volumes de estoque de materiais e medicamentos e na determinação do preço de venda do serviço.

A gestão de custos em um hospital necessita estar alinhada às estratégias da entidade, visto que os registros dos gastos vão servir de instrumento de acompanhamento dos serviços, e com base na estratégia, uma possível redefinição das prioridades essenciais como aumento da produtividade e racionalização do uso de recursos, dentre outras medidas administrativas, podem ser tomadas (OLIVEIRA et al., 2007).

As particularidades da atividade hospitalar, aliada à complexidade dos serviços prestados, bem como sua diversidade, tornam o processo de apuração dos custos nestas instituições uma tarefa que exige um excelente sistema de custeio, apoiado por um sistema de informação bem gerenciado. Para Matos (2002), no que diz respeito à gestão hospitalar, o sistema de gerenciamento por centro de custos é o mais viável, isto porque ele disponibiliza informações sobre o quanto foi gasto e indica a quais centros de custos se vinculam os gastos do hospital. 
Diante disso, o presente estudo busca investigar: como é feita a apuração de custos por centro de custos nos diversos setores da um hospital de grande porte na Zona da Mata mineira? Objetivou-se com esse estudo, identificar como são apurados os custos dos serviços prestados em um hospital de grande porte pelo método do centro de custos. Especificamente, objetivou-se identificar quais os centros de custos criados para a apuração dos custos, bem como os procedimentos utilizados para apuração destes, apresentando inclusive as dificuldades durante o processo.

Alguns estudos sobre custos em hospitais já foram desenvolvidos no Brasil nos últimos anos, como de Abbas, Lezana e Menezes (2002) que utilizaram o método $\mathrm{ABC}$ no serviço de processamento de roupas de um hospital, Souza et al. (2013) que calculam os custos de procedimentos de urologia com base no custeio ABC e comparam com os valores repassados pelo SUS, Raupp, Crispim e Almeida (2007) que analisaram o processo de gestão de custos em uma maternidade, Abbas e Leoncine (2014) que apresentam o cálculo do parto cesárea em seis hospitais que utilizam o método de custeio das seções homogêneas.

Com base nos estudos apresentados, o presente estudo faz-se relevante em detrimento à carência de trabalhos que apresentem o desenvolvimento e a execução de um sistema de apuração de custos pelo método do centro de custos em uma organização hospitalar, apresentando assim a operacionalidade do sistema, bem como as dificuldades detectadas durante este processo. O estudo se justifica também pela necessidade de se obter uma ferramenta que viabilize o equilíbrio financeiro da organização de saúde, por meio da administração dos custos, despesas e receitas, para que esta sobreviva ao mercado competitivo e ofereça serviços ao nível de excelência.

\section{Gestão de Custos em Instituições de Saúde}

A preocupação decorrente das dificuldades de gestão dos recursos econômicos e financeiros dos hospitais está relacionada com a sobrevivência da instituição, bem como a realização de seus objetivos, crescimento e consolidação no mercado; sendo assim, a gestão de custos é um instrumento fundamental, como fonte de informação para tomada de decisões e identificação de possíveis desvios rumo à realização de metas traçadas.

De modo geral, a apuração de custos parece fácil, pois significa calcular todos os recursos consumidos pela instituição e distribuí-los de acordo com os serviços executados. Porém, a complexidade dos recursos utilizados, a existência dos custos das áreas de apoio (administrativas ou não produtivas), entre outros, torna essa ação mais difícil.

\section{Conceito de Custos Hospitalares}

Para Martins (2000), os custos são gastos medidos em termos monetários, incorridos ou potencialmente a incorrer, que viabilizam o serviço médico, ou seja, os custos de um hospital são gastos para a operacionalização de suas atividades e prestação de serviços. Segundo o mesmo autor, os custos podem ser diretos ou indiretos, fixos e variáveis.

Bertó e Beulke (2000) definem custos diretos, como custos possíveis de identificar diretamente sua unidade de serviço ou procedimento, bem como calcular com precisão o custo do procedimento, item da tabela etc. Como exemplo: consumo de medicamentos, órtese, prótese por conta, consumo de materiais médico-hospitalar por paciente; formação de custos financeiros relativos à concessão de prazos e convênios etc.

Ainda com base nos mesmos autores, os custos indiretos são aqueles comuns a vários procedimentos, não podem ser calculados individualmente por item de serviço prestado, bem como tendem a apresentar maior complexidade de mensuração. Podem ser: energia elétrica, água, seguro, depreciação etc.

Para Martins (2000), os custos fixos são aqueles que não se alteram ao longo da produção médica e a responsabilidade de sua inocorrência 
cabe à alta administração; por outro lado, os custos variáveis são aqueles que variam na mesma proporção do volume de produção médica (pacientes atendidos e com alta no período). Geralmente os custos hospitalares diretos são também classificados como variáveis e os custos indiretos, como fixos.

Visto isso, tem-se que o custo total de um hospital é soma dos custos indiretos e custos diretos, conforme apresentado na Figura 1 a seguir.

Figura 1 - Custo hospitalar total.

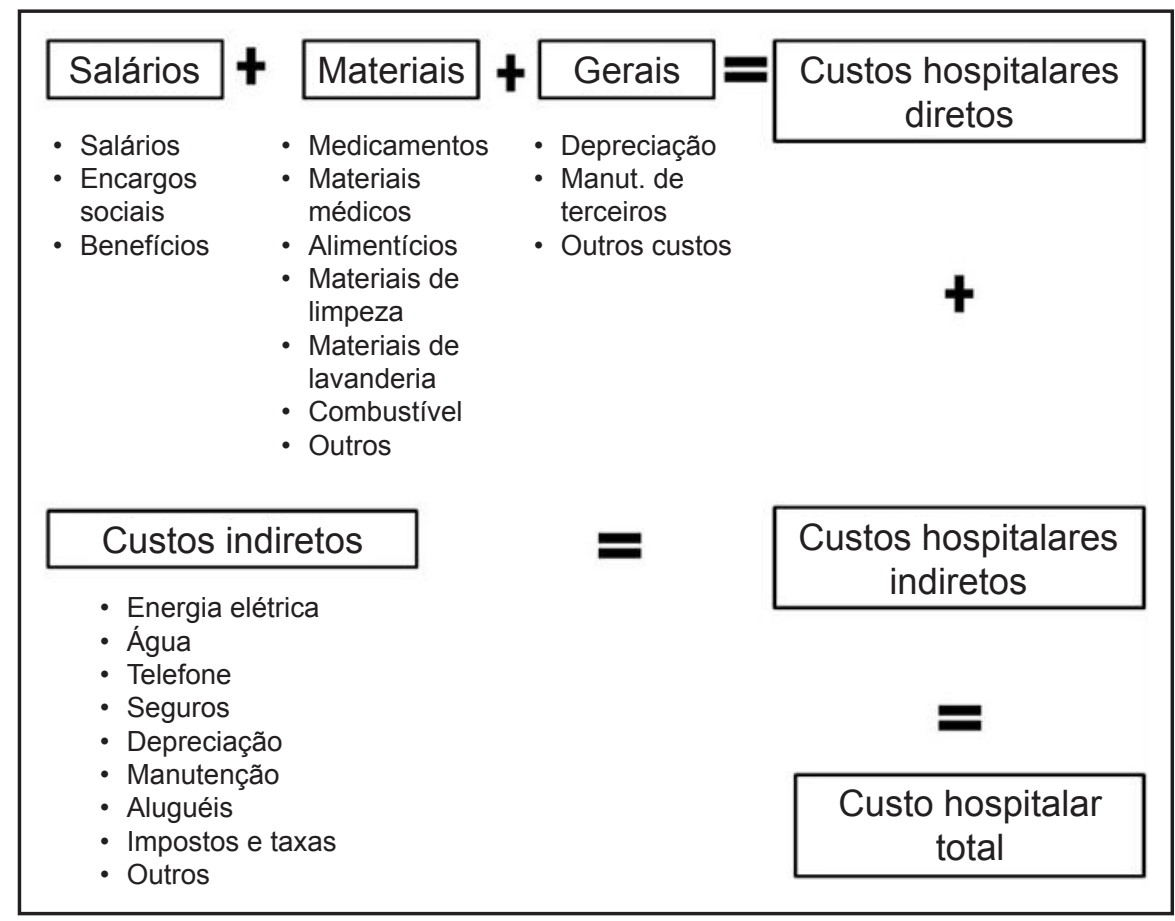

Fonte: Martins (2000)

Para Bonacim e Araújo (2010) a apuração de custos em hospitais é uma atividade abrangente, levando em conta a diversidade dos serviços prestados, o que exige inicialmente uma delimitação dos sistemas de custos e um sistema de informações eficiente.

Diante da complexidade que envolve toda atividade hospitalar, a alocação dos custos indiretos se torna uma atividade que exige a implantação de um sistema de custos para controlar os resultados, maximizar os lucros, sem afetar a qualidade dos serviços. Segundo Oliveira et al. (2007), os sistemas mais comuns para administrar os custos hospitalares são: por centro de custos e por atividade.

O método do centro de custos, criado na Europa no início do século XX, é o mais utilizado no mundo, segundo Perez Junior, Oliveira e Costa (2009). Sendo assim, esse será o foco desse trabalho.

\section{Sistema de Custeio por Absorção}

Conforme Camargos e Gonçalves (2004), no sistema de custeio por absorção todos os custos de produção (diretos ou indiretos) são alocados aos produtos. Inicialmente, classificam-se os custos em indiretos e diretos, sendo que ambos são "absorvidos" pelos produtos/serviços durante o processo de produção. Desta forma, faz a apropriação somente dos custos, sendo que as despesas (diretas ou indiretas) não são atribuídas aos produtos e serviços, e sim lançadas em sua totalidade na demonstração do resultado do exercício (DRE).

Leão e Riccio (2000) apresentam as etapas do método de custeio por absorção, onde primeiro se classificam os gastos em custos e despesas, em seguida se apropriam os custos diretos ao produto/ serviço e depois se atribui aos custos indiretos um 
critério de rateio previamente estabelecido. Bonacim e Araújo (2010) afirmam que a determinação do critério de rateio deve estar baseada no bom senso dos gestores para que se tenha uma apropriação mais real e justa possível.

Martins (2003) afirma que o custeio por absorção é o sistema utilizado obrigatoriamente pela legislação brasileira, porém com algumas exceções. Esta obrigatoriedade força as organizações a adotarem, caso desejarem, outro sistema de custeio de maneira paralela. Além dos obstáculos iniciais incorridos em qualquer mudança ou implantação de metodologia de trabalho, essa situação torna-se um empecilho, considerada a necessidade de aumento dos recursos disponíveis para a manutenção de dois sistemas de custeio.

Falk (2001) afirma que, no que tange a gestão de custos em hospitais, a criação e identificação dos centros de custos são de vital importância para o processo de apuração dos custos. Bertó e Beulke (2000) definem centro de custo como unidades homogêneas em termos de responsabilidade, fonte geradora de custos, identidade de objetivos, existência de unidade de mensuração específica etc.

Conforme Raupp, Crispim e Almeida (2007), o sistema de gestão por centro de custo varia de um estabelecimento para outro, pois nem todos se destinam ao mesmo tipo de atendimento, além de haver diferenciações de recursos tecnológicos. Apesar disso, ainda segundo os mesmos autores, algumas características e preocupações devem estar presentes em qualquer gestão. Para eles, o acompanhamento do dinamismo da instituição, impondo acréscimos, junções ou supressões de centro de custos, para acompanhar a realidade da instituição em cada momento, é fundamental, além, é claro, da classificação e codificação dos centros de custos, que deve ser uma prática habitualmente usada para facilitar a sua identificação.

Martins (2000) destaca que o sistema de custos hospitalares deve refletir a forma dos procedimentos médicos, métodos da produção médica e a maneira com que os itens de custos são consumidos pela produção médica.

Para Abbas e Leoncine (2014), os serviços hospitalares são realizados em unidades, seções, setores etc., que pela complexidade da atividade, recebem custos diretos, indiretos e transferências intersecionais, motivo pelo qual é utilizado o método de cálculo de custo por setor, ou centro de custos.

No Quadro 1, são apresentados os departamentos produtivos, apoio e administrativos e seus respectivos centros de custos. Segundo Bertó e Beulke (2000) este é um modelo padrão para ser aplicado em um hospital.

Quadro 1 - Departamentalização e centro de custos.

\begin{tabular}{|c|c|c|}
\hline \multicolumn{2}{|c|}{ Departamentos } \\
\hline Produtivos & Apoio & Administrativos \\
\hline Serviços médicos & Higiene e limpeza & Administração \\
\hline Enfermagem & Lavanderia & Contabilidade \\
\hline Berçário & Hotelaria & Controladoria \\
\hline Maternidade & Manutenção & Diretoria \\
\hline Serv. de urgência e emergência & Vigilância & \\
\hline Clínica Médica & Pessoal & \\
\hline Cardiologia & Centro de esterilização & \\
\hline CTls & Nutrição & \\
\hline Farmácia & Ambulância & \\
\hline Radiologia & & \\
\hline
\end{tabular}

Fonte: Bertó e Beulke (2000) 
Segundo Rocchi (1982), os centros de custos produtivos são centros geradores de receita, ou seja, correspondem aos centros de custos que prestam ou fornecem atendimento e serviços diretamente aos pacientes e representam a atividade-fim do hospital, como, por exemplo, o Centro Cirúrgico, as Unidades de Internamento e Radiologia.

Os Centros Auxiliares ou de Apoio correspondem aos centros cuja principal função é a de auxiliar o funcionamento de outros centros de custo e/ou prestarem serviços para todo o estabelecimento, como, por exemplo, os Serviços de Vigilância, de Portaria e de Limpeza, afirma Rocchi (1982). De acordo com o mesmo autor, os centros administrativos correspondem aos centros de custos responsáveis pelos trabalhos de supervisão, controle e informação, como, por exemplo, a Contabilidade, a Gerência e o Financeiro.

\section{Cálculo dos Custos}

A metodologia de apuração de custos proposta por Palácio (1993) diz que para se fornecer o custo total dos serviços prestados a cada paciente, os custos devem seguir um roteiro sucessivo de alocações e discriminações. O primeiro passo é a apuração do custo total de cada centro de custos, onde são alocados os custos aos diversos Centros de Custos. Para os custos indiretos, sugere-se utilizar mapas próprios para fazer esta alocação. Obtido o custo total de cada setor, faz se o rateio dos custos dos centros que prestam serviços para os centros que recebem estes serviços, como, por exemplo, o custo total da lavanderia deve ser repassado para os centros que se utilizam das roupas lavadas.

A segunda etapa do rateio de custos preconiza a divisão dos custos de cada centro para as unidades de serviços geradas naquele setor. Para isso é necessário que cada Centro Gerador de Receita defina qual é a unidade de serviço que ele presta. Assim, o Centro Radiológico pode determinar que a sua base de rateio será o número de radiografias; os postos de enfermagem e a UTI, número de diárias; e assim sucessivamente.
Segundo Palácio (1993), a determinação exata do custo de cada serviço só é possível ao final de cada período mensal. Isso exige que o hospital se utilize de sistemas de custo-padrão, no qual o total de custos de cada centro, bem como o número total de unidades de serviços a serem produzidas no período, sejam previstos e depois comparados com o efetivo. Eventuais variações, tanto credoras quanto devedoras, podem ser alocadas diretamente para a conta de resultados.

Segundo Martins (2000), a determinação dos custos hospitalares por prontuário ou ordem de produção médica é o sistema empregado por todos os hospitais. Nesse processo, cada prontuário é considerado um procedimento, no qual materiais médicos, medicamentos, taxas, salários, encargos sociais, benefícios, gêneros alimentícios, energia elétrica, água, telefone, entre outros, são atribuídos de forma direta ou por rateios. Cada paciente deve ter o seu prontuário individual, onde são lançados todos os gastos e requisições médicas. À medida que os pacientes recebem alta, os valores de produção médica são lançados em folhas de custos, que calculados mensalmente, compõem os custos hospitalares mensais.

A partir dos resultados obtidos com a apuração dos custos mensais, as gerências poderão tomar decisões mais assertivas.

\section{Procedimentos Metodológicos}

No intuito de identificar como são apurados os custos dos serviços prestados por um hospital de grande porte na Zona da Mata mineira a pesquisa se utiliza de abordagem qualitativa e descritiva. Na visão de Marconi e Lakatos (2017) a pesquisa qualitativa possui como premissa a análise e interpretação dos aspectos mais densos, de modo a descrever a complexidade da realidade e do comportamento humano e ainda busca subsidiar análises mais detalhadas sobre as investigações, atitudes e tendências de comportamento. Neste sentido, buscou-se conhecer a realidade do hospital em análise de maneira aprofundada por meio dos gestores responsáveis pela gestão de custos da 
entidade. Já a abordagem descritiva na percepção de Gil (2010) tem o intuito de descrever os fatos e fenômenos de determinada realidade. Assim, a realidade a ser descrita neste estudo é a maneira como são apurados os custos em um hospital tendo por base a sistemática de centro de custos.

A pesquisa adota a ferramenta de estudo de caso como forma de observar as práticas de gestão de custos adotadas. O caso objetiva analisar sob a perspectiva dos gestores os principais fatores que determinam as formas de alocação de custos no hospital e as possíveis dificuldades diante da complexidade da realidade de uma organização hospitalar de grande porte. Para Yin (2015), o estudo de caso representa uma investigação empírica e compreende um método abrangente, com a lógica do planejamento, da coleta e da análise de dados. Gil (2010) completa explicando que o estudo de caso deve ser realizado com perguntas simples, objetivas e claras.

A escolha do caso segue as recomendações de Eisenhardt (1989) em relação à amostragem teórica, na qual a escolha seria feita de forma que o caso reúna as características que necessitariam ser observadas para o objetivo da pesquisa. Dessa forma, selecionou-se uma organização hospitalar de grande porte que apresentasse unidades de atendimentos diferenciadas que pudessem ser analisadas como centros de custos. Além disso, a escolha também é justificada pela acessibilidade, dado o interesse dos gestores em participar e ao fato de um dos pesquisadores ter acesso às informações e fontes de evidência.

Para execução do estudo de caso foram utilizadas três estratégias de coletas de dados: pesquisa documental, observação direta e entrevista semiestruturada. A pesquisa documental consiste na consulta de fontes documentais diversificadas e dispersas em relação aos dados que se pretende analisar, que ainda não sofreram tratamento analítico, tais como: tabelas estatísticas, relatórios, documentos oficiais, relatórios de empresas, dentre outros (MARCONI; LAKATOS, 2017).

Na visão de Martins e Theóphilo (2016) as técnicas observacionais podem ser consideradas procedimentos empíricos de natureza sensorial. Nesta linha, na observação direta o pesquisador se torna parte integrante da realidade no intuito de coletar informações, dados e evidências para o estudo.

A última forma de coleta de evidências foi a entrevista semiestruturada, que possibilita identificar as informações desejadas, com espaço para captar outros detalhes do contexto de interesse (BRYMAN, 2012). A coleta de dados direcionada à composição de uma visão geral da empresa e a forma de alocação dos custos ocorreu no mês de julho de 2017. As informações de custeio são referentes ao mês de janeiro de 2017.

Para o tratamento dos dados foram utilizadas as transcrições da entrevista, as anotações da observação direta e o software Microsoft Excel ${ }^{\circledR}$ para a organização das informações financeiras. Os resultados são apresentados de forma descritiva e utiliza de elementos visuais como gráficos, figuras e tabelas com o intuito de facilitar o entendimento.

\section{Objeto de estudo}

A entidade que serviu de objeto de estudo para essa pesquisa é um hospital de grande porte da Zona da Mata mineira. A instituição foi fundada há mais de 150 anos e realiza cerca de 18 mil procedimentos cirúrgicos por ano, pois concilia tradição, filantropia, modernização e qualidade no atendimento. A entidade mantenedora do hospital é uma entidade sem fins lucrativos e associada à Igreja Católica.

O hospital é administrado por um conselho de administração, composto por 15 membros efetivos e cinco suplentes, sendo obrigatoriamente um terço de médicos, e um representante da Arquidiocese. Em 2008, criou-se um estatuto onde se estabeleceu a gestão executiva da instituição, composta pelos cargos: gestor geral e administrativo, gestor financeiro, diretor clínico, diretor técnico e chefe de enfermagem, todos remunerados, contratados pelo conselho de administração.

Por ser uma entidade filantrópica, o hospital não tem finalidade lucrativa, e as fontes de recursos 
para a sua manutenção são provenientes dos atendimentos aos pacientes do Sistema Único de Saúde-SUS, de convênios e também da remuneração dos pacientes via atendimento particular.

\section{Estudo de caso}

O sistema de custos utilizado atualmente no hospital é o por centro de custos e foi estabelecido em 2008, quando foi implantada uma política de custo juntamente com um Sistema de Gestão de Saúde parametrizado e integralizado, e, para tanto, um setor exclusivo foi criado com intuito de administrar as receitas e as despesas da instituição, o que acaba por proporcionar aos pacientes/ clientes um serviço de qualidade sem comprometer o resultado financeiro.
Para atender aos conceitos do sistema por centro de custos, a apuração destes seguem algumas etapas até se chegar ao custo total dos serviços prestados ao paciente, ou seja, até a soma dos custos diretos, que são os custos possíveis de identificar diretamente na sua unidade de serviço ou no procedimento, e os custos indiretos, que são aqueles comuns a vários procedimentos e não podem ser calculados individualmente por item de serviço prestado, bem como tendem a apresentar maior complexidade de mensuração (BERTÓ; BEULKE, 2000).

A identificação e a classificação dos centros de custo ou setores e das contas de custo, tiveram como base a atividade exercida; desta forma, os centros de custo e as contas que o compõe são apresentados no Quadro 2 a seguir:

Quadro 2 - Centros de custo por grupo de centro de custos.

\begin{tabular}{|c|c|c|c|}
\hline \multicolumn{4}{|c|}{ Centros de custo por grupo de centro de custos } \\
\hline Administrativo & Apoio / Auxiliares & Intermediário & Produtivos \\
\hline Administração & Alimentação e Nutrição & Centro Epidem., Estat. Pesquisa & Ambulatório Clín. Especializado \\
\hline Assessoria de Comunicação & Almoxarifado & Centro de Imagem & Centro Obstétrico \\
\hline Assessoria Jurídica & Arquitetura e Engenharia & Comitê de Ética em Pesquisa & CTI Cirúrgico \\
\hline Auditoria Interna & $\mathrm{CCIH}$ & Endoscopia & CTI Geral Adulto \\
\hline Chefia de Enfermagem & Centro de Estudos & Hemodinâmica & CTI Infantil \\
\hline Contabilidade & CIHDOTT - Doação de Órgãos & Laboratório de Análises Clínicas & Espaço Clínico \\
\hline Coord. Técnica Enfermagem & CME - Centro Esterilização & Neurofisiologia Clínica & Farmácia \\
\hline Custos e Orçamentos & Condomínio Geral & Radiologia & Serviço de Urg. Especializada \\
\hline Desenvolvimento Humano & Depreciação & Triagem Auditiva Neonatal & U.I. Neonatologia \\
\hline Direção Clínica & Engenharia Clínica & $\mathrm{UCC} 1^{\circ}$ andar & U.I. $10^{\circ}$ andar \\
\hline Direção de Ensino & Eto Esterilização & $\mathrm{UCC} 14^{\circ}$ andar & U.I. $11^{\circ}$ andar \\
\hline Direção Técnica & Fisioterapia & Ultrimagem & U.I. $12^{\circ}$ andar \\
\hline Educação - NES & Higienização e Limpeza Hosp. & Unidade Transfusional & U.I. $2^{\circ}$ andar \\
\hline Estudos e Projetos & Hospitalistas & & U.I. $3^{\circ}$ andar \\
\hline Ger. Comercial e Faturamento & Manutenção Geral & & U.I. $4^{\circ}$ andar \\
\hline Financeiro / Tesouraria & Núcleo Segurança do Paciente & & U.I. $5^{\circ}$ andar \\
\hline Gerenciamento de Leitos & Órtese e Prótese & & U.I. $6^{\circ}$ andar \\
\hline Hotelaria & Processamento de Roupas & & U.I. $7^{\circ}$ andar \\
\hline Licitação e Compras & Residência Médica & & U.I. $8^{\circ}$ andar \\
\hline Meio ambiente & SAME & & U.I. $9^{\circ}$ andar \\
\hline Ouvidoria & Serv. Apoio Aleit. Mater. - SAAM & & Unidade Coronária $5^{\circ}$ andar \\
\hline Patrimônio & Time de Resposta Rápida & & Unidade de Pós-angioplastia \\
\hline Pessoal & & & UPI Cirurgia Geral \\
\hline Presidência & & & UPI Ortopedia \\
\hline Psicologia Hospitalar & & & UPI Transplantes \\
\hline
\end{tabular}


Continuação

\begin{tabular}{|l|l|l|l|}
\hline Qualidade & & & \\
\hline Recepção / Internação & & & \\
\hline Recrutamento e Seleção & & & \\
\hline Repasse Médico & & & \\
\hline Segurança Corporativa & & & \\
\hline Seg. Med. Trabalho - SESMT & & & \\
\hline Serviço Social / Ouvidoria & & & \\
\hline Tecnologia da Informação & & & \\
\hline
\end{tabular}

Fonte: Autores

As contas distribuídas dentro de cada centro de custos foram agrupadas quanto ao tipo de gasto, ou seja, se elas se referem a uma despesa com pessoal, administrativa, financeira, operacional etc.

Após calculado o custo total por centro de custos e geral do hospital, é preciso fazer o rateio dos custos dos centros que prestam serviços para os centros que recebem estes serviços, como, por exemplo, o custo total da lavanderia deve ser repassado para os centros que se utilizam das roupas lavadas.

O sistema de rateio e apropriação dos custos indiretos do hospital é composto de três mapas, que distribuem os custos em cascata, fazendo uma alocação sequencial. Para Falk (2001), no processo de alocação sequencial reúnem-se os custos totais do primeiro centro e por meio de um critério dividemse os custos aos demais centros. O total de custos do primeiro centro torna-se zero e os demais centros de custos absorvem este montante proporcionalmente ao critério estabelecido anteriormente. O processo se repete em cada centro auxiliar até que todos estes tenham o montante de seus custos zerados e apenas os centros produtivos possuam custos.

No estudo em questão, os centros Administrativos e Auxiliares são os primeiros a terem seus gastos "distribuídos" aos centros de custos Intermediário e Produtivos, e na sequência, o centro de custo Auxiliar passará pelo mesmo processo. Esta sequência de rateios permite que ao final seja calculado o custo paciente/dia por centro de custo, conforme apresentado na Figura 2 a seguir.

Figura 2 - Processo de rateio.

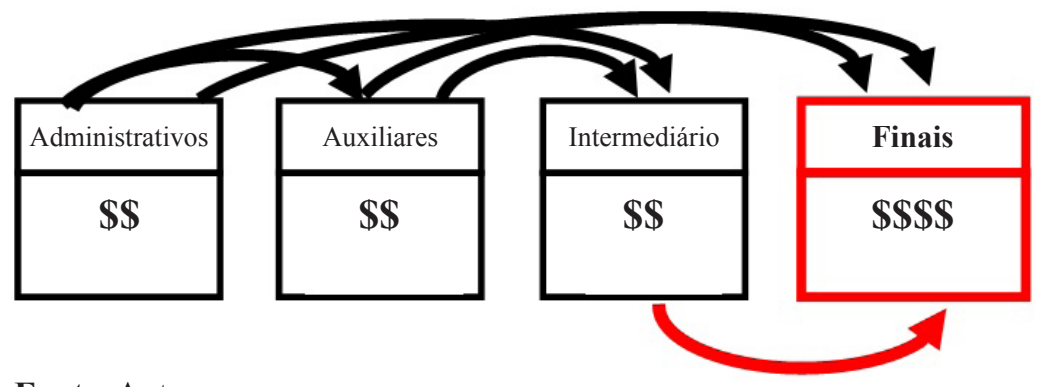

Fonte: Autores

Para que sejam realizados os rateios dos centros de custos Administrativos e Auxiliares, o setor de custos utiliza três mapas que irão balizar o processo. O primeiro mapa distribui os gastos dentro do próprio centro de custos em sete grupos: consumo de materiais, despesas administrativas, despesas com pessoal, despesas financeiras, despesas operacionais, honorários/repasse e impostos e taxas. As informações apresentadas são baseadas no mês de janeiro de 2017 .

Com base nesta classificação, os custos totais distribuídos nos quatro centros de custo são apresentados no Quadro 3 a seguir: 
Quadro 3 - Custos totais por centro de custos.

\begin{tabular}{|l|r|r|r|r|r|}
\hline Grupo de Conta de Custo & Administrativo & Apoio / Auxiliares & Intermediário & Produtivos & Custo Total \\
\hline Consumo Mat. / Med. & $3.149,88$ & $58.558,47$ & $1.785 .620,83$ & $1.181 .687,49$ & $3.029 .016,67$ \\
\hline Despesas Administrativas & $785.782,14$ & $399.222,60$ & $442.401,25$ & $227.955,04$ & $1.855 .361,03$ \\
\hline Despesas com Pessoal & $1.059 .307,07$ & $1.065 .610,72$ & $776.387,79$ & $2.128 .036,10$ & $5.029 .341,68$ \\
\hline Despesas Financeiras & $1.290,61$ & - & - & - & $1.290,61$ \\
\hline Despesas Operacionais & $14.492,68$ & $158.061,49$ & $312.247,12$ & $160.551,90$ & $645.353,19$ \\
\hline Honorários / Repasse & 808,20 & $191.476,70$ & $398.885,67$ & $896.693,25$ & $1.487 .863,82$ \\
\hline Impostos e Taxas & $1.823,80$ & $6.292,67$ & - & - & $8.116,47$ \\
\hline Total & $\mathbf{1 . 8 6 6 . 6 5 4 , 3 8}$ & $\mathbf{1 . 8 7 9 . 2 2 2 , 6 5}$ & $\mathbf{3 . 7 1 5 . 5 4 2 , 6 6}$ & $\mathbf{4 . 5 9 4 . 9 2 3 , 7 8}$ & $\mathbf{1 2 . 0 5 6 . 3 4 3 , 4 7}$ \\
\hline
\end{tabular}

Fonte: Autores

Gráfico 1 - Custo total.

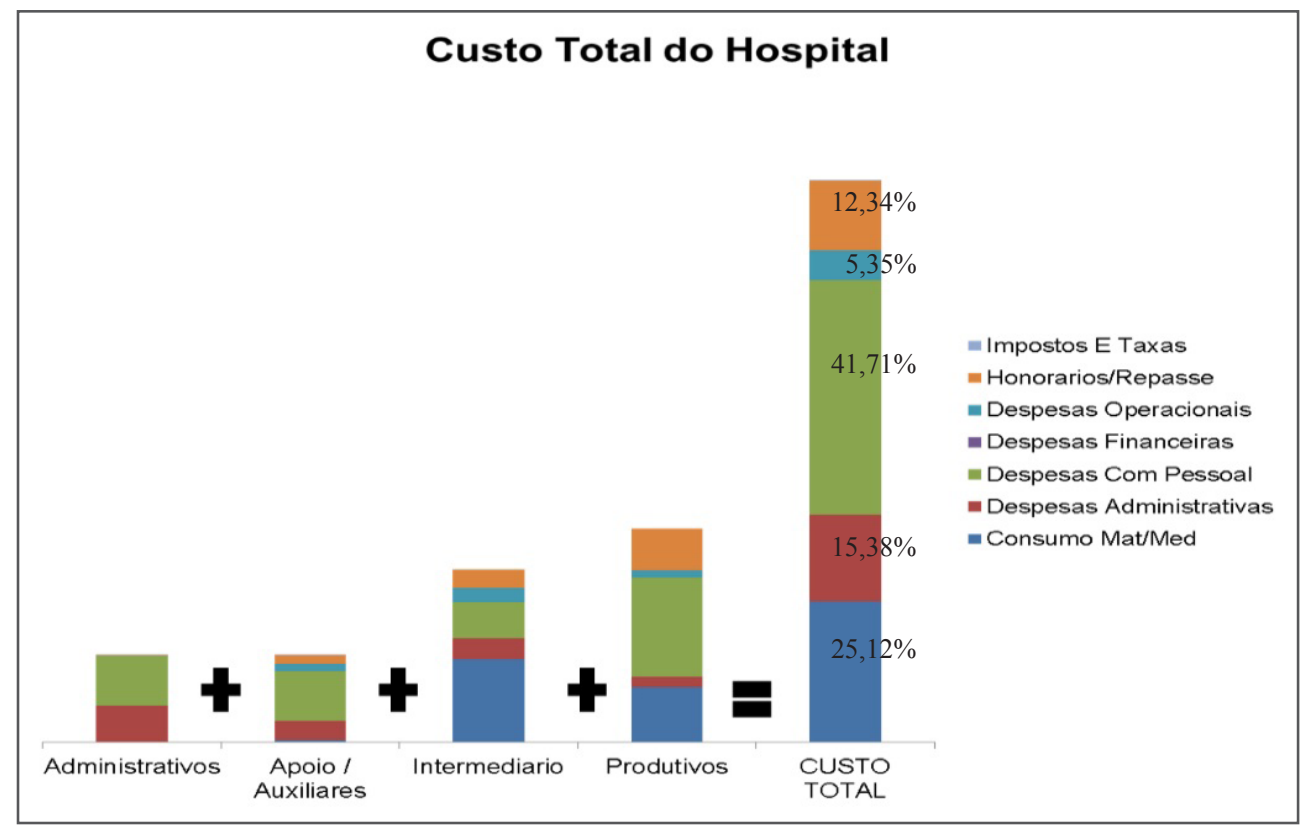

Fonte: Autores

O segundo mapa irá ratear os custos dos Centros de Custo Administrativos e Auxiliares para determinação do custo das unidades de serviço, e o terceiro mapa realiza o rateio dos Centros de Custo Intermediários (unidades de serviço) para determinação do custo do paciente-dia.

O critério de rateio é baseado na unidade de mensuração ou indicadores, onde se verifica a abrangência, e o valor a ratear, e este é dividido proporcionalmente aos setores da abrangência de acordo com os montantes informados para a unidade de mensuração. Caso não tenha abrangência, o valor a ratear é dividido proporcionalmente aos montantes informados para cada centro de custo da unidade de mensuração em questão.

As unidades de mensuração com as quais se determinará o custo unitário de cada centro de custos são definidas de acordo com a atividade do setor e suas particularidades, e o rateio é feito proporcionalmente à sua produção. Como exemplo: para os custos do setor de Higiene e Limpeza o rateio é realizado baseado no metro quadrado; no setor de 
Psicologia Hospitalar o indicador é o número de atendimentos; Processamento de roupas, obtido por quilo de roupa lavada. Nos centros de custos onde não se identifica unidades de mensuração tão facilmente são utilizados o rateio por incidência percentual, que consiste em ratear o valor total do centro de custo proporcionalmente com o valor do custo primário dos setores informados na abrangência.

Os Quadros 4 e 5 apresentam de forma simplificada o rateio dos centros de custos administrativos e apoio/auxiliares aos centros produtivos e intermediários.

Quadro 4 - Rateio do centro Administrativos aos demais centros.

\begin{tabular}{|l|r|r|r|r|r|}
\hline Grupo de Conta de Custo & \multicolumn{1}{c|}{ Administrativos } & \multicolumn{1}{c|}{ Apoio / Auxiliares } & \multicolumn{1}{c|}{ Intermediário } & \multicolumn{1}{c|}{ Produtivos } & \multicolumn{1}{c|}{ Total } \\
\hline Consumo Mat. / Med. & $3.149,88$ & $58.558,47$ & $1.785 .620,83$ & $1.181 .687,49$ & $4.027 .935,09$ \\
\hline Despesas Administrativas & $785.782,14$ & $399.222,60$ & $442.401,25$ & $227.955,04$ & $2.007 .697,62$ \\
\hline Despesas com Pessoal & $1.059 .307,07$ & $1.065 .610,72$ & $776.387,79$ & $2.128 .036,10$ & $4.021 .934,10$ \\
\hline Despesas Financeiras & $1.290,61$ & - & - & - & $182.780,51$ \\
\hline Despesas Operacionais & $14.492,68$ & $158.061,49$ & $312.247,12$ & $160.551,90$ & $5.010 .240,32$ \\
\hline Honorários / Repasse & 808,20 & $191.476,70$ & $398.885,67$ & $896.693,25$ & $4.456 .147,98$ \\
\hline Impostos e Taxas & $1.823,80$ & $6.292,67$ & - & - & $15.617,01$ \\
\hline Total & $\mathbf{1 . 8 6 6 . 6 5 4 , 3 8}$ & $\mathbf{1 . 8 7 9 . 2 2 2 , 6 5}$ & $\mathbf{3 . 7 1 5 . 5 4 2 , 6 6}$ & $\mathbf{4 . 5 9 4 . 9 2 3 , 7 8}$ & $\mathbf{1 2 . 0 5 6 . 3 4 3 , 4 7}$ \\
\hline Rateio setor Administrativo & $-1.866 .654,38$ & $83.999,45$ & $587.996,13$ & $1.194 .658,80$ & - \\
\hline Soma & - & $\mathbf{1 . 9 6 3 . 2 2 2 , 1 0}$ & $\mathbf{4 . 3 0 3 . 5 3 8 , 7 9}$ & $\mathbf{5 . 7 8 9 . 5 8 2 , 5 8}$ & $\mathbf{1 2 . 0 5 6 . 3 4 3 , 4 7}$ \\
\hline
\end{tabular}

Fonte: Autores

Quadro 5 - Rateio do centro Apoio / Auxiliares aos demais centros.

\begin{tabular}{|l|r|r|r|r|r|}
\hline Grupo de Conta de Custo & \multicolumn{1}{|c|}{ Administrativos } & \multicolumn{1}{c|}{ Apoio / Auxiliares } & \multicolumn{1}{c|}{ Intermediário } & \multicolumn{1}{c|}{ Produtivos } & \multicolumn{1}{c|}{ Total } \\
\hline Consumo Mat. / Med. & $3.149,88$ & $58.558,47$ & $1.785 .620,83$ & $1.181 .687,49$ & $4.027 .935,09$ \\
\hline Despesas Administrativas & $785.782,14$ & $399.222,60$ & $442.401,25$ & $227.955,04$ & $2.007 .697,62$ \\
\hline Despesas com Pessoal & $1.059 .307,07$ & $1.065 .610,72$ & $776.387,79$ & $2.128 .036,10$ & $4.021 .934,10$ \\
\hline Despesas Financeiras & $1.290,61$ & - & - & - & $182.780,51$ \\
\hline Despesas Operacionais & $14.492,68$ & $158.061,49$ & $312.247,12$ & $160.551,90$ & $5.010 .240,32$ \\
\hline Honorários / Repasse & 808,20 & $191.476,70$ & $398.885,67$ & $896.693,25$ & $4.456 .147,98$ \\
\hline Impostos e Taxas & $1.823,80$ & $6.292,67$ & - & - & $15.617,01$ \\
\hline Total & $\mathbf{1 . 8 6 6 . 6 5 4 , 3 8}$ & $\mathbf{1 . 8 7 9 . 2 2 2 , 6 5}$ & $\mathbf{3 . 7 1 5 . 5 4 2 , 6 6}$ & $\mathbf{4 . 5 9 4 . 9 2 3 , 7 8}$ & $\mathbf{1 2 . 0 5 6 . 3 4 3 , 4 7}$ \\
\hline Rateio setor Administrativo & $-1.866 .654,38$ & $83.999,45$ & $587.996,13$ & $1.194 .658,80$ & - \\
\hline Soma & - & $\mathbf{1 . 9 6 3 . 2 2 2 , 1 0}$ & $\mathbf{4 . 3 0 3 . 5 3 8 , 7 9}$ & $\mathbf{5 . 7 8 9 . 5 8 2 , 5 8}$ & $\mathbf{1 2 . 0 5 6 . 3 4 3 , 4 7}$ \\
\hline Rateio setor Apoio / Auxiliares & - & $-1.963 .222,10$ & $655.716,18$ & $1.307 .505,92$ & - \\
\hline Soma & - & - & $\mathbf{4 . 9 5 9 . 2 5 4 , 9 7}$ & $\mathbf{7 . 0 9 7 . 0 8 8 , 5 0}$ & $\mathbf{1 2 . 0 5 6 . 3 4 3 , 4 7}$ \\
\hline
\end{tabular}

Fonte: Autores

Segundo Coura et al. (2009), o método de apropriação em cascata permite que cada centro de custo transfira, por rateio, seu custo total a todos os centros de custos que lhe sucedam no plano hierarquizado de centros de custo. Com isso, pode haver a transferência de custo também entre centros de custo do mesmo grupo onde ocorra troca de serviços. Por esse sistema, os centros 
de custo que já tiveram seus custos rateados, não receberão rateios dos demais centros, o processo se repete até que os centros produtivos tenham absorvidos todos os custos.

Dessa forma todos os custos gerados das operações do hospital são absorvidos nos centros de custos produtivos e intermediários e agregados aos valores cobrados dos pacientes/clientes. O terceiro mapa apresenta a apropriação final aos centros de custos intermediários e produtivos em suas contas. O Quadro 6 apresenta os custos apropriados dentro do centro de custos intermediário.

Quadro 6 - Centro de custos Intermediário.

\begin{tabular}{|l|r|r|r|}
\hline $\begin{array}{c}\text { Centros de Custo de Rateio / } \\
\text { Centros de Custos }\end{array}$ & $\begin{array}{c}\text { Custo Primário }+ \\
\text { Produto Direto }\end{array}$ & Total Rateios & $\begin{array}{c}\text { Custo Operacional } \\
\text { Final }\end{array}$ \\
\hline Intermediário & $\mathbf{3 . 7 1 5 . 5 4 2 , 6 6}$ & $\mathbf{1 . 2 4 3 . 7 1 2 , 3 1}$ & $\mathbf{4 . 9 5 9 . 2 5 4 , 9 7}$ \\
\hline Centro Epidem., Estat. Pesquisa & $17.780,27$ & $3.952,40$ & $21.732,67$ \\
\hline Centro de Imagem & $10.660,37$ & $3.204,78$ & $13.865,15$ \\
\hline Comite de Ética em Pesquisa & 150,10 & $1.009,02$ & $1.159,12$ \\
\hline Endoscopia & $44.161,08$ & $33.907,01$ & $78.068,08$ \\
\hline Hemodinâmica & $501.058,56$ & $116.539,59$ & $617.598,15$ \\
\hline Laboratório de Análises Clínicas & $477.777,90$ & $107.123,51$ & $584.901,41$ \\
\hline Neurofisiologia Clínica & $6.900,81$ & $6.009,07$ & $12.909,88$ \\
\hline Radiologia & $213.294,16$ & $65.583,10$ & $278.877,27$ \\
\hline Triagem Auditiva Neonatal & $1.920,53$ & 772,79 & $2.693,32$ \\
\hline UCC $1^{\circ}$ andar & $248.344,60$ & $150.499,71$ & $398.844,31$ \\
\hline UCC 14 ${ }^{\circ}$ andar & $1.998 .010,61$ & $722.746,13$ & $2.720 .756,74$ \\
\hline Ultrimagem & $112.321,92$ & $10.144,60$ & $122.466,51$ \\
\hline Unidade Transfusional & $83.161,77$ & $22.220,59$ & $105.382,36$ \\
\hline
\end{tabular}

Fonte: Autores

Com base no Quadro 6, tem-se então o custo operacional de cada um dos setores intermediários e estes custos são a base para se calcular o custo dos serviços prestados por esses setores.

No Quadro 7, são apresentados os custos operacionais do setor produtivo.

Quadro 7 - Centro de custos Produtivos.

\begin{tabular}{|l|r|r|r|}
\hline $\begin{array}{c}\text { Centros de Custo de Rateio / } \\
\text { Centros de Custos }\end{array}$ & $\begin{array}{c}\text { Custo Primário }+ \\
\text { Produto Direto }\end{array}$ & Total Rateios & $\begin{array}{c}\text { Custo Operacional } \\
\text { Final }\end{array}$ \\
\hline Produtivos & $\mathbf{4 . 5 9 4 . 9 2 3 , 7 8}$ & $\mathbf{2 . 5 0 2 . 1 6 4 , 7 2}$ & $\mathbf{7 . 0 9 7 . 0 8 8 , 5 0}$ \\
\hline Ambulatório Clín. Especializado & $16.481,17$ & $8.419,47$ & $24.900,64$ \\
\hline Centro Obstétrico & $73.302,19$ & $35.684,50$ & $108.986,68$ \\
\hline CTI Cirúrgico & $278.478,21$ & $136.248,08$ & $414.726,28$ \\
\hline CTI Geral Adulto & $480.033,94$ & $227.333,04$ & $707.366,98$ \\
\hline CTI Infantil & $362.980,91$ & $169.611,13$ & $532.592,04$ \\
\hline Espaço Clínico & $236.648,32$ & $96.185,72$ & $332.834,03$ \\
\hline Farmácia & $188.535,96$ & $95.647,11$ & $284.183,07$ \\
\hline Serviço de Urg. Especializada & $708.660,43$ & $319.686,82$ & $1.028 .347,24$ \\
\hline U.I. Neonatologia & $102.743,32$ & $56.213,22$ & $158.956,54$ \\
\hline U.I. $10^{\circ}$ andar & $158.294,50$ & $111.940,75$ & $270.235,25$ \\
\hline
\end{tabular}


Continuação

\begin{tabular}{|l|r|r|r|}
\hline U.I. $11^{\circ}$ andar & $174.245,86$ & $114.090,22$ & $288.336,08$ \\
\hline U.I. $12^{\circ}$ andar & $113.734,13$ & $84.548,56$ & $198.282,69$ \\
\hline U.I. $2^{\circ}$ andar & $191.076,23$ & $114.577,22$ & $305.653,45$ \\
\hline U.I. $3^{\circ}$ andar & $100.433,76$ & $75.264,61$ & $175.698,37$ \\
\hline U.I. $4^{\circ}$ andar & $122.223,58$ & $91.826,30$ & $214.049,88$ \\
\hline U.I. $5^{\circ}$ andar & $130.964,22$ & $95.704,45$ & $226.668,67$ \\
\hline U.I. $6^{\circ}$ andar & $111.493,87$ & $83.890,09$ & $195.383,96$ \\
\hline U.I. $7^{\circ}$ andar & $190.316,21$ & $118.727,40$ & $309.043,61$ \\
\hline U.I. $8^{\circ}$ andar & $108.323,88$ & $78.983,98$ & $187.307,86$ \\
\hline U.I. $9^{\circ}$ andar & $228.406,79$ & $140.980,84$ & $369.387,63$ \\
\hline Unidade Coronária $5^{\circ}$ andar & $237.716,75$ & $116.585,51$ & $354.302,26$ \\
\hline Unidade de Pós-angioplastia & $16.450,98$ & $9.577,97$ & $26.028,95$ \\
\hline UPI Cirurgia Geral & $91.363,10$ & $40.713,42$ & $132.076,52$ \\
\hline UPI Ortopedia & $87.022,87$ & $42.920,12$ & $129.942,99$ \\
\hline UPI Transplantes & $84.992,61$ & $36.804,21$ & $121.796,82$ \\
\hline
\end{tabular}

Fonte: Autores

O resultado dos custos diretos somados ao cálculo dos custos indiretos por centro de custos proporciona aos gestores o acompanhamento dos custos totais por período ou competência, que são comparados a períodos anteriores do mesmo ano e ao mesmo período em anos anteriores, possibilitando o gerenciamento dos custos, a avaliação da produtividade e a comparação com os valores de receita obtida.

O sistema de custo implantado no hospital tem como objetivo à determinação dos preços dos serviços, assim como uma análise interna que permita um melhor conhecimento dos resultados da gestão e uma melhoria no funcionamento e na qualidade do atendimento na organização.

\section{Considerações Finais}

A implantação de um sistema de gestão de custos eficiente em instituições de saúde é essencial para gerenciamento dos resultados e tomada de decisões, porém, definir o sistema de custeio que será usado e reunir os dados necessários para isto é uma tarefa complexa em se tratando de um hospital. Muito se discute a respeito do melhor método de custeio para organizações hospitalares, e conclui-se que, apesar de não ser o mais apropriado, o método de centro de custo é considerado o mais viável.

O presente estudo evidencia a aplicação da gestão de custos em uma organização hospitalar, conhecendo o sistema de custos de um hospital da Zona da Mata mineira, que utiliza como principal método de custeio, o custeio por absorção e faz a apropriação dos custos por centros de custos.

Dentro da atual estrutura, são apresentados os quatro centros de custos que o hospital foi dividido: administrativo, apoio/auxiliar, intermediário e produtivo, e as contas/atividades que integram cada uns desses centros. Apresentase ainda os rateios realizados, apropriando os valores dos centros de custos administrativo e apoio/auxiliar aos centros intermediários e produtivos. Nesses últimos, são apresentados os valores recebidos de cada um dos rateios nas suas diversas contas/atividades.

O hospital utiliza os mapas de apropriação, que são três, para o gerenciamento dos custos e também para definir de forma eficiente os critérios para a distribuição dos custos indiretos dos centros de custos administrativos e apoio/auxiliar, para os intermediários e produtivos. 
Diante do que foi exposto, o presente estudo atinge seus objetivos e recomenda-se que esta pesquisa seja realizada em outras empresas do segmento, a fim de identificar qual sistema de custo é utilizado para o gerenciamento dos custos e quais as dificuldades encontradas para a gestão dos recursos financeiros, dada a complexidade da organização.

\section{Referências}

ABBAS, K.; LEONCINE, M. Cálculo dos custos dos procedimentos médicos hospitalares em hospitais brasileiros. RAHIS: Revista de Administração Hospitalar, Belo Horizonte, v. 11, n. 1, p. 1-11, jan. /mar. 2014.

ABBAS, K.; LEZANA, Á. G. R.; MENEZES, E. A. Apuração dos custos nas organizações hospitalares: o método $\mathrm{ABC}$ aplicado no serviço de processamento de roupas de um hospital. Revista da FAE, Curitiba, v. 5, n. 2, p.77-97, maio/ ago. 2002.

BAUMGARTNER, R. R. Avaliação $d a$ aplicabilidade do custeio $A B C$ : activity based costing na acurácia de custos na área hospitalar, especificamente na Unidade de Terapia Intensiva: Estudo de um caso prático. 1998. Dissertação (Mestrado em Ciências Financeiras e Contábeis) Pontifícia Universidade Católica de São Paulo, São Paulo, 1998.

BERTÓ, D.; BEULKE, R. Gestão de custos e resultados na saúde. 2. ed. São Paulo: Saraiva, 2000 .

BONACIM, C. A. G.; ARAÚJO, A. M. P. Gestão de custos aplicada a hospitais universitários públicos: a experiência do Hospital das Clínicas da Faculdade de Medicina de Ribeirão Preto da USP. Revista de Administração Pública, Rio de Janeiro, v. 44, n. 4, p. 903-931, 2010.

BORBA, V. R. Administração hospitalar: princípios básicos. 3. ed. São Paulo: Cedas, 1991.

BRYMAN, A. Social research methods. 4. ed. Oxford: Oxford University Press, 2012.
CAMARGOS, M. A.; GONÇALVES, M. A. Sistemas de acumulação de custos, métodos de custeio, critérios de atribuição de custos e tipos de custo: uma diferenciação didático-teórica para o ensino da disciplina Contabilidade de Custos. Revista ANGRAD, Rio de Janeiro, v. 6, n. 1, p. 97118, 2004.

COURA, B.; PINTO, A. A. G.; SALGADO, F. F.; DANTAS, M. B. Gestão de custos em saúde. Rio de Janeiro: Ed. FGV, 2009.

DALLORA, M. E. R. V. Gerenciamento de custos de material de consumo em um hospital de ensino. 2007. Dissertação (Mestrado em Saúde na Comunidade) - Faculdade de Medicina, Universidade de São Paulo, Ribeirão Preto, SP, 2007.

EISENHARDT, K. M. Building theories from case study research. Academy of Management Review, Ada, v. 14, n. 4, p. 532-550, 1989.

FALK, J. A. Gestão de custos para hospitais: conceitos, metodologias e aplicações. São Paulo: Atlas, 2001.

GIL, A. C. Como elaborar projetos de pesquisa. 5. ed. São Paulo: Atlas, 2010.

LEÃO, L. C. G.; RICCIO, E. L. A determinação do custo médio ponderado do capital em condições de risco. In: CONGRESSO BRASILEIRO DE CUSTOS, 8., 2000. Recife. Anais [...]. Recife: Associação Brasileira de Custos, 2000. Disponível em: https://bit.ly/2Z812jA. Acesso em: 3 mar. 2017.

MARCONI, M.A.; LAKATOS, E. M. Fundamentos de metodologia cientifica. 8. ed. São Paulo: Atlas, 2017.

MARTINS, D. Custos e orçamentos hospitalares. São Paulo: Atlas, 2000.

MARTINS, E. Contabilidade de custos. São Paulo: Atlas, 2003.

MARTINS, G. A.; THEÓFILO, C. R. Metodologia da investigação cientifica para ciências sociais aplicadas. 3. ed. São Paulo: Atlas, 2016. 
MATOS, A. J. Gestão de custos hospitalares: técnicas, análise e tomada de decisão. São Paulo: Editora STS, 2002.

NEGRA, C. A. S.; NEGRA, E. M. S. Custo hospitalar: uma reflexão sobre implantação e necessidades. Contabilidade Vista e Revista, Belo Horizonte, v. 12, n. 1, p. 31-56, 2001.

OLIVEIRA, A. D. S.; BRUNI, A. L.; PAIXÃO, R. B.; CARVALHO JUNIOR, C. V. Estratégia, custos e hospitais: um diagnóstico na cidade de Salvador, Bahia (2006). In: CONGRESSO BRASILEIRO DE CUSTOS, 14., 2007, João Pessoa. Anais [...]. Recife: Associação Brasileira de Custos, 2007. Disponível em: https://bit.ly/3byg3hi. Acesso em: 3 mar. 2017.

PALÁCIO, E. L. Dinâmica de custos em empresas hospitalares: um estudo de caso. Enfoque: Reflexão Contábil, Maringá, n. 6, p. 19-24, 1993.

PEREZ JUNIOR, J. H.; OLIVEIRA, L. M.; COSTA, R. G. Gestão estratégica de custos. 6 . ed. São Paulo: Atlas, 2009.

RAUPP, F. M.; CRISPIM, C. H.; ALMEIDA, É. S. Gestão de custos hospitalares por meio do custeio por absorção: o caso da maternidade Carmela Dutra. RIC: Revista de Informação Contábil, Recife, v. 2, n. 1, p. 120-133, out. /dez. 2007.

ROCCHI, C. A. Apuração de custos em estabelecimentos hospitalares. Revista Brasileira de Contabilidade, Brasília, n. 41, p. 19-27, 1982.

SOUZA, A. A.; XAVIER, A. G.; LIMA, L. C. M.; GUERRA, M. Análise de custos em hospitais: comparação entre os custos de procedimentos de urologia e os valores repassados pelo sistema único de saúde. ABCustos, São Leopoldo, v. 8, n. 1, p. 92-109, jan. /abr. 2013.

YIN, R. K. Estudo de caso: planejamento e métodos. 5. ed. Porto Alegre: Bookman, 2015. 
